\title{
The Application of Maglev Supporting Technology in NC Machine
}

\author{
Zhongqiao Zheng ${ }^{1,2, *}$, Xiaojing Wang ${ }^{1}$, Jiansheng Zhang ${ }^{2}$ and Yanhong Zhang ${ }^{2}$ \\ ${ }^{1}$ School of Mechatronic Engineering and Automation, Shanghai University, Shanghai, 200072, China; \\ ${ }^{2}$ School of Electronic Information \& Electric Engineering, Changzhou Institute of Technology, Changzhou, 213002, \\ China
}

\begin{abstract}
Feed motion guides driven by linear motor in NC machine for industrial applications mostly adopt mechanical support and they easily produce wear because of impact and high speed. For above characteristic and the requirement of machining accuracy, application of maglev supporting technology in NC Machine is raised. By analyzing the structure of maglev feed motion guide, the mechanical model of single-degree-freedom and multi-degree-freedom magnetic suspension system is proposed. By MATLAB, the curve of stiffness and damper in steady state is gained from the two groups of PID parameters, so that the influence of the controller parameters on the stiffness and damper is obtained and the vibration area of feed motion guides is made smaller. The conclusion can be reached that the performance of maglev feed motion guides is optimized by adjusting the parameters of controller properly that has an important theoretical significance for improving machining accuracy of NC machine tools.
\end{abstract}

Keywords: Control parameters, feed motion guides, linear motor, maglev, NC machine.

\section{INTRODUCTION}

At present, feed motion guides are driven by linear motor in $\mathrm{NC}$ machine for industrial applications. Mostly NC machines adopt mechanical support technology, such as rolling guide, V-V sliding guide, hydrostatic guide-way, aerostatic guide-way, etc. In above, the first two types of guide-way easily produce wear and creep because of impact and high speed during high-acceleration and high-speed motion. The latter two types belong to contactless guide-way in which the former is characterized by a bigger hydraulic device, more ancillary equipments, difficult maintenance of oil ducts and serious oil pollution and the latter requires keeping the environment so clean that machining is difficult $[1,2]$.

With the rapid development of maglev support technology, it is expected to apply magnetic levitation to feed motion guides driven by linear motor in NC machine as maglev trains that has a high-precision positioning requirement. At present there are no reports about the applications of maglev feed motion guide that is made possible.

Compared with traditional machine guide-way, maglev guide-way is characterized by no mechanical friction, no wear, no lubrication, there is no creeping phenomenon, no decreasing precision and life problem because of wear and contact fatigue [3]. Its reliability is better than traditional guide-ways because the reliability of electronic components is better than of mechanical parts [4]. It is without pollution because there are no large oil auxiliary equipments and does not require super-clean dust-proof environment because its precision requirements are lower than aerostatic guide-way's. As active controlling is used, information processing capability of machine tool is improved [5]. Maglev guide-way has a wider operating temperature range, easier maintenance, longer life. So maglev support technology has developed very fast world-wide.

\section{STRUCTURE OF MAGLEV LINEAR FEED MO- TION GUIDE}

Maglev linear guide-way supports the feed guide-way by using magnetic force. The entire system fully achieves frictionless movement by applying maglev support technology to change the guide-way of feed mechanism from contact support to non-contact support and using non-contact linear motor to drive and improve its feed precision. Overall performance of the system has a direct relationship with structure design of maglev linear feed guide-way which is affected by many factors including the loads size, direction, property and distribution. The number of electromagnetics, their size and installation locations. The location and installation method of linear motor and the gap between feed motion guide way and linear guide way. guide-way The feed motion guide-way is composed of base, support electromagnet, motor, feed platform, guidance electromagnet and linear guideway. Four pair of support electromagnet and two pair of guidance electromagnet are installed on the guide-way, on which sensors are directly installed. Adjustment pad is made of non- magnetic conductive stainless steel, which has two functions: the first is adjusting the gap to $0.2 \mathrm{~mm}$ between electromagnet and linear guide-way; the second is fluxinsulation which prevents leakage flux along feed motion 


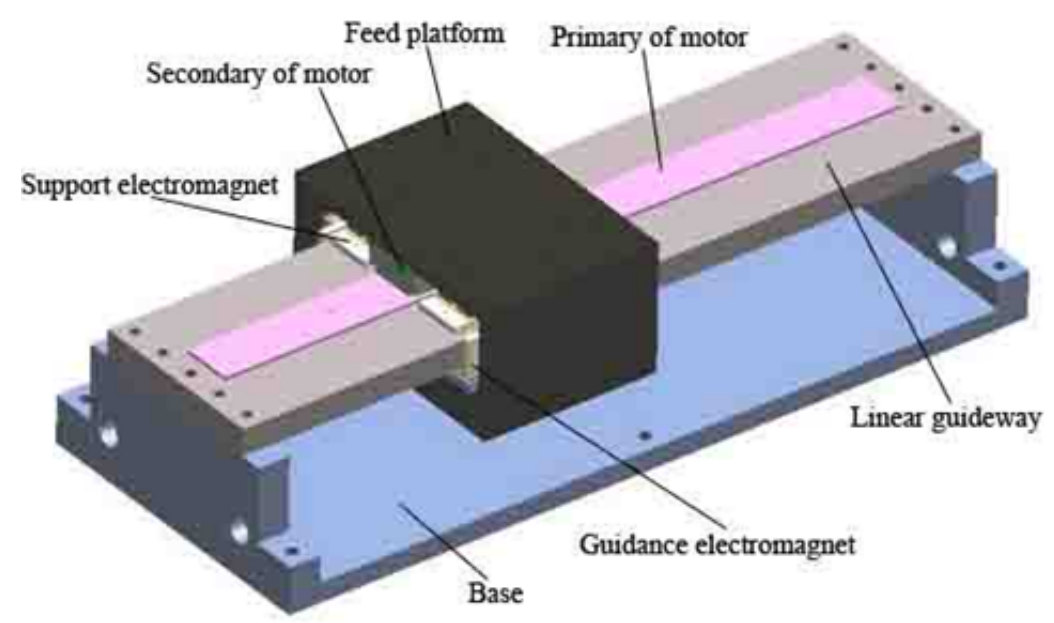

Fig. (1). The structure of maglev linear feed platform system.

guide-way. The magnetic force that support electromagnets produce hold feed motion guide-way in suspension, which maintains a gap of $0.2 \mathrm{~mm}$. Guidance electromagnets supply sufficient horizontal guidance force to prevent contact between guide-ways when feed guide-way is subjected to a transverse force. The secondary of linear motor is installed below feed guide-way, and the primary is installed on linear guide-way, between which the gap of $0.5 \sim 0.8 \mathrm{~mm}$ is maintained. Linear motor non-contact drives the secondary with maglev guide-way to move without friction along linear guide-way under precise control. The role of linear guideway is to support feed guide-way to move. The structure of maglev linear feed motion guide-way is shown in Fig. (1). Displacement sensor measures the change of the gap between two guide-ways to output an electrical signal, from which the controller derives the control signal. The control signal is transformed to control current by amplifier to be sent to the electromagnet. So magnetic force is changed to maintain the guide-way at equilibrium position in [6].

The linear guide-way is an important component of maglev feed motion guide system, whose material must have better permeability from electromagnetic and higher mechanical strength, such as the 10\# steel and A3 steel, which can be used as the material of linear guide-way, of course, industrial pure iron can also be used. In the range of $0 \sim 1.2 \mathrm{~T}$, magnetization characteristics curve of these materials is linear, basically. A3 steel was first used as the material for the linear guide-way, but the experimental results suggested that dimensional accuracy of A3 steel has a larger change with time. 45\# medium carbon steel is adequate for such a role, which provides convenience for engineering applications. Considering from support precision, the surface of guideway towards sensor requires higher machining precisions because support precision depends on feedback signal from sensors of control system, if the surface is rough, there is a deviation in feedback signal from sensors which reduces support precision.

Active magnetic suspension support technology is used to realize support without friction, improve support stiffness and damping of the guide-way, prevent resonance at the critical frequency while in operation, decrease mechanical vi- bration and ensure stability of system operation in high speed, which must have enough loading ability and dynamic stiffness to ensure reliability of system operation. For designing the support electromagnet, the static loading capacity requirement is first considered and dynamic stiffness is ensured by control system [7].

Maglev feed motion guide-way uses four pairs of electromagnets to load and two couples to guide whose iron cores are made from industrial pure iron in a $U$ shape. When current passed through the coil, magnetic force is produced that levitates the feed motion guide-way and sustains load. After the material is determined, static working point $B_{0}$ of magnetic induction intensity is selected at the midpoint of linear segment in magnetization characteristics curve.

The structure of an electromagnet is shown as Fig. (2). whose parameters are as following:

Material of iron core: industrial pure iron

Static working point: $B_{0}=0.55 \mathrm{~T}$

Gap between electromagnet and guide-way: $c_{0}=0.2 \mathrm{~mm}$

Ratio of auxiliary supporting gap to magnetic supporting gap: $\eta_{c}=0.5$

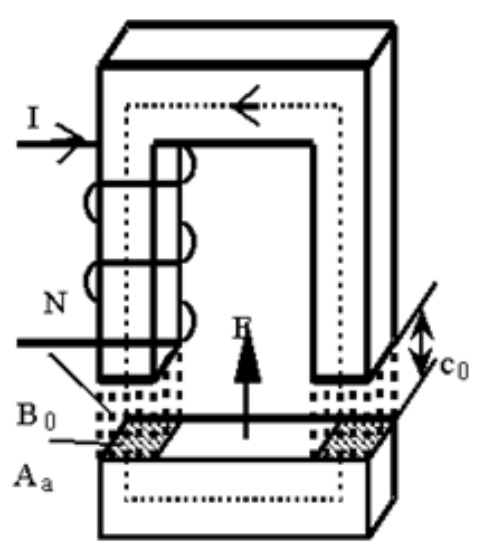

Fig. (2). Basic structure of an electromagnet. 


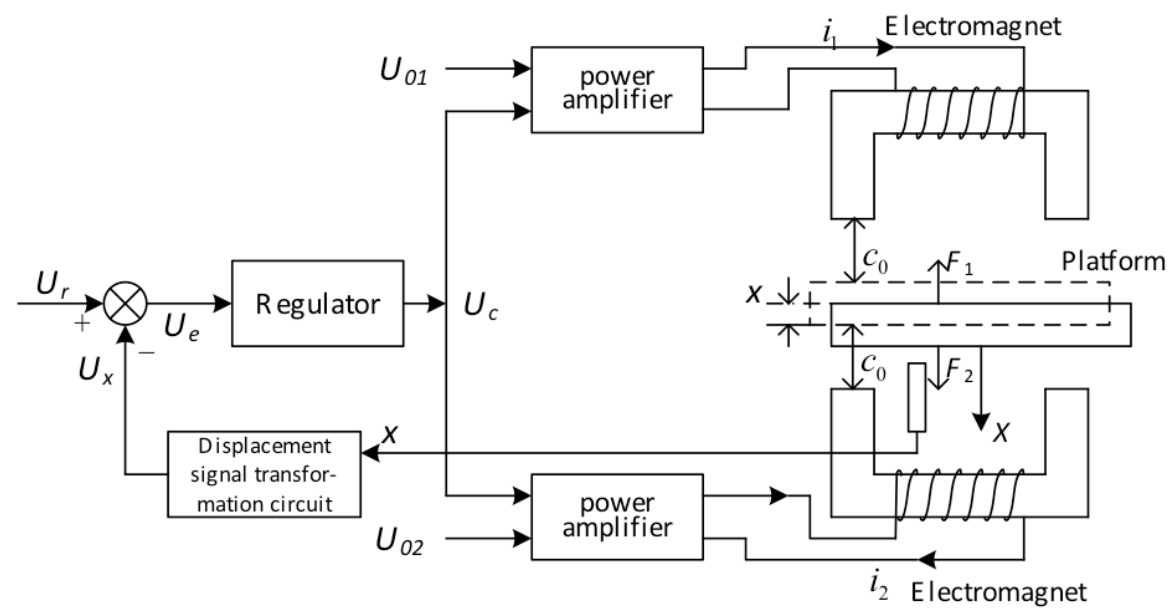

Fig. (3). Schematic diagram of single-degree-freedom maglev support system.

Parameters of support electromagnet are as following: $45 \mathrm{~mm}$

Shape of electromagnet: Length $a=20 \mathrm{~mm}$, Width $b=$

Area of magnetic pole: $A_{a}=a b=0.0009 \mathrm{~m}^{2}$

Bearing capacity: $F_{0}=385.2 \mathrm{~N}$

Bearing capacity of four couples electromagnets: $F=4 F_{0}$ $=1540.8 \mathrm{~N}$

Bias current: $I_{0}=1.0 \mathrm{~A}$

Number of turns: $N=240$

Current density: $j=3 \mathrm{~A} / \mathrm{m}^{2}$

Dual-coil structure is used in which double-winding is used, so $N_{1}=60$, including five layers composed of 12 turns. Due to the factors of mutual inductance and iron core, actual measured value of coil inductance is $0.58 \mathrm{mH}$.

Parameters of guidance electromagnet are as following:

Dimensions of electromagnet: Length $a=10 \mathrm{~mm}$, Width $b=40 \mathrm{~mm}$

Area of magnetic pole: $A_{a}=a b=0.0009 \mathrm{~m}^{2}$

Bearing capacity: $F_{0}=171.3 \mathrm{~N}$

Bearing capacity of two couples electromagnets: $F=2 F_{0}$ $=342.6 \mathrm{~N}$

Bias current: $I_{0}=1.0 \mathrm{~A}$

Number of turns: $N=220$

Actual measured value of coil inductance: $0.44 \mathrm{mH}$.

\section{MATHEMATICAL MODEL OF SINGLE-DEGREE- FREEDOM MAGLEV SUPPORT SYSTEM}

Single-degree-freedom maglev support system is composed of upper and lower electromagnets, and it keeps balance by adjusting magnetic force based on sensor's feedback, whose schematic diagram is shown as Fig. (3). Physical meaning of each value in Fig. (3) is as following:

$c_{0}$ : gap between electromagnet and linear guide-way as equilibrium
$U_{01}, U_{02}$ : voltage that bias current of upper and lower magnetic coil require respectively

$x$ : displacement of guide center in the degree of freedom

$U_{x}$ : output voltage of signal conversion circuit corresponding to $x$

$U_{r}$ : reference voltage as guide lays in center position

$U_{e}$ : voltage of error signal

$U_{c}$ : output voltage of regulator

$i_{1}, i_{2}$ : respective current of two magnetic coils including bias current $I_{0}$ and control current $i_{\mathrm{x}}$

$F_{1}, F_{2}$ : magnetic force that two electromagnets produce respectively

Position of guide-way can be precisely controlled by controller composed of displacement sensor, signal conversion circuit, regulating circuit and power amplifier. When guide center has a deviation $x$ from equilibrium position, signal conversion circuit converts displacement signal detected by sensor to voltage signal which is compared to reference voltage $U_{r}$ for error signal $U_{e}$. From $U_{\mathrm{e}}$, regulator derives voltage signal $U_{\mathrm{c}}$, which is converted to control current by power amplifier to adjust magnetic force of electromagnet. In such a way, the guide-way is steadily at equilibrium position.

Electromagnet is of current superposition that control current $i_{x}$ and bias current $I_{0}$ use one coil. Guide-way whose mass is $\mathrm{m}$ is placed between upper electromagnet I and lower electromagnet II. Assumptions are made as following:

(1) Neglecting leakage flux of the winding;

(2) Neglecting reluctance of core and guide-way;

(3) Neglecting hysteresis loss and eddy-current loss of magnetic material.

Resultant force of upper and lower electromagnet on the guide-way is zero at equilibrium position. When a disturbance makes the guide-way deviate away from the equilibrium position, the downward offset is $\mathrm{x}$ shown as Fig. (3), and to make the guide-way go back to its equilibrium position, a control current $i_{x}$ must be added to make electromagnetic force of upper electromagnet larger and electromagnetic 
force of lower electromagnet smaller. The electromagnetic force of both of electromagnet respectively are

$$
\begin{aligned}
& F_{1}=\frac{\mu_{0} S_{0} N^{2}\left(I_{0}+i_{x 0}+i_{x}\right)^{2}}{4\left(c_{0}+x\right)^{2}} \\
& F_{2}=\frac{\mu_{0} S_{0} N^{2}\left(I_{0}-i_{x 0}-i_{x}\right)^{2}}{4\left(c_{0}-x\right)^{2}}
\end{aligned}
$$

Resultant force on the guide-way is

$$
\Delta F_{x}=F_{1}-F_{2}=\frac{\mu_{0} S_{0} N^{2}}{4}\left[\left(\frac{I_{0}+i_{x 0}+i_{x}}{c_{0}+x}\right)^{2}-\left(\frac{I_{0}-i_{x 0}-i_{x}}{c_{0}-x}\right)^{2}\right]
$$

Where, the meanings of the symbols are as follows:

$\mu_{0}-$ air permeability of ferromagnetic materials $\left(4 \pi \times 10^{-7} \mathrm{H} / \mathrm{m}\right)$

$S_{0}$ - total pole face area

$N$ - number of turns per coil

$I_{0}$ - bias current of the magnetic coil

$i_{x}$ - control current

$i_{x 0}$ - current used to offset static load at the direction of $X$

$c_{0}$ - nominal air gap

$x$ - displacement of the guide-way center

As the guide-way is at the equilibrium position $(x=0$, $i=0$ ), the static load at the direction of $X$ is

$$
F_{x 0}=\frac{\mu_{0} S_{0} N^{2}}{4}\left[\left(\frac{I_{0}+i_{x 0}}{c_{0}}\right)^{2}-\left(\frac{I_{0}-i_{x 0}}{c_{0}}\right)^{2}\right]
$$

So

$i_{x 0}=\frac{c_{0}^{2} F_{x 0}}{\mu_{0} S_{0} N^{2} I_{0}}$

Neglecting quadratic term and higher order term of $i$ and $x$, the Taylor series expansion of the equation (4) can be obtained near the equilibrium point $x=0, i=0$ as follows:

$$
F_{x 0}=\frac{\partial F}{\partial x} \cdot x+\frac{\partial F}{\partial i} \cdot i_{x}=k_{x x 0} x+k_{i x 0} i_{x}
$$

Where,

$$
k_{x x 0}=-\frac{\mu_{0} S_{0} N^{2}\left(I_{0}^{2}+i_{x 0}^{2}\right)}{c_{0}^{3}} \text { - displacement stiffness coeffi- }
$$

cient

$$
k_{x i 0}=\frac{\mu_{0} S_{0} N^{2} I_{0}}{c_{0}^{2}}-\text { voltaic stiffness coefficient }
$$

$k_{x x 0}$ and $k_{x i 0}$ are related to the structure and working point of electromagnet. So $k_{x x 0}$ and $k_{x i 0}$ are constants after the structure and working point of electromagnet are determined. Based on Newton's Second Law, neglecting other forces on the guide-way, mechanics equation in the direction of $X$ is $m \ddot{x}+k_{x x 0} x+k_{i x 0} i_{x}=f_{x}$

\section{ELECTRIC EQUATION OF SINGLE-DEGREE- FREEDOM MAGLEV SUPPORT SYSTEM}

Electric equation of the system is the balance equation between voltage or current signal of electromagnet coils and output signal of controller. In order to make analysis simple, it is considered that two electromagnets are structurally symmetrical and offset $x$ is positive as the guide-way moves down.

The voltage $u_{L}$ of coils can be expressed as following:

$u_{L}=u_{1}-u_{2}=2 i_{x} R_{0}+\frac{d \Psi_{1}}{d t}-\frac{d \Psi_{2}}{d t}$

Where,

$R_{0}-$ DC resistance of single control coils

$u_{1}, u_{2}$ - voltages of two coils

$\Psi_{1}, \Psi_{2}$ - total flux linkage of two iron cores, $\Psi_{1}=L_{1} \times i_{1}$, $\Psi_{2}=L_{2} \times i_{2}$

As neglecting leakage flux of the winding and reluctance of core and guide-way, because length of air gap is $2 x$, inductance is as following:

$L=\frac{\mu_{0} S_{0} N^{2}}{2 x}$

Putting the relevant equations into equation (8), the following equationcan be obtained:

$$
\begin{aligned}
u_{L}= & 2 i_{x} R_{0}-\frac{\mu_{0} S_{0} N^{2}}{2}\left[\frac{I_{0}+i_{c}}{\left(c_{0}+x\right)^{2}}+\frac{I_{0}-i_{c}}{\left(c_{0}-x\right)^{2}}\right] \dot{x} \\
& +\frac{\mu_{0} S_{0} N^{2}}{2}\left[\frac{1}{c_{0}+x}+\frac{1}{c_{0}-x}\right] \dot{i}_{x}
\end{aligned}
$$

Set output voltage of power amplifier to $u_{o c}$, based on the voltage balance, the following equation can be obtained:

$u_{L}=u_{o c}$

The signal that power amplifier provides to coils of stator can be current or voltage. Supposing input voltage of power amplifier is $u_{c}$, and gain coefficient is $\lambda$, then

$u_{o c}=\lambda u_{c}$

Supposing output current of power amplifier is $i_{x}$, mutual conductance coefficient is $\beta$, and transfer function is irrelevant to load characteristics, then

$i_{x}=\beta u_{c}$

The above analysis proves that there are completely different effects as electric equation of the system uses different control variable (current or voltage). Here, the system composed of equation (8), (12) and (8), (13) respectively is called mathematical model of voltage control and voltage control. 


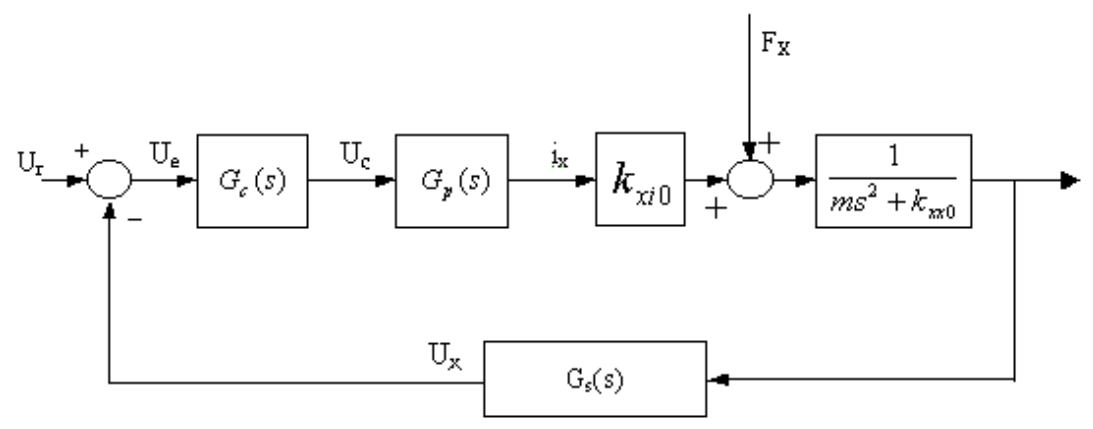

Fig. (4). Transfer function block diagram of maglev supporting system.

\section{STIFFNESS AND DAMPING CHARACTERISTICS OF MAGLEV SUPPORTING SYSTEM}

Dynamic characteristics of maglev supporting system mainly depends on its stiffness and damping characteristics which are two key parameters of mechanical vibration analysis, and concern stability, vibration, anti-jaming capability, and so on. Stiffness of maglev supporting system can be classified into passive stiffness depending on structural feature and active stiffness depending on controller, the latter of which is numerically much larger and changes with change of controller parameters and plays a good role in regulating dynamic performance of the system.

Using Laplace transformation for (7), the following equation can be obtained

$m s^{2} X(s)=k_{x x 0} X(s)+k_{x i 0} I_{x}(s)+F_{x}(s)$

Where, $X(s), I_{x}(s), F_{x}(s)$ are the Laplace transformation of $x(t), i_{x}(t)$ and $f_{x}(t)$, respectively.

Supposing $G_{s}(s), G_{c}(s)$ and $G_{p}(s)$ are the transfer function of displacement sensor, regulator and power amplifier, respectively and the reference input signal is zero, closed loop block diagram of the system shown in Fig. (4) can be obtained.

Supposing $G(s)=G_{s}(s) \bullet G_{c}(s) \bullet G_{p}(s)$.

$G(s)$ is the transfer function of total controller; the closed loop transfer function is as following

$$
X(s)=\frac{F_{x}(s)}{m s^{2}+k_{x x 0}+k_{x i 0} G(s)}
$$

Setting $s=j \omega$, then

$$
X(j \omega)=\frac{F_{x}(j \omega)}{\left[k_{x i 0} \operatorname{Re}(G(j \omega))+k_{x x o}-m \omega^{2}\right]+j\left[k_{x i 0} \operatorname{Im}(G(j \omega))\right]}
$$

Based on mechanics principle, motion equation of second order mechanical system is as following

$$
m \ddot{x}+c \dot{x}+k x=F_{x}
$$

Where, $m, c, k$ represent mass, damping and stiffness respectively. The following equation can be obtained using Laplace transformation for (17)

$$
m s^{2} X(s)+c s X(s)+k X(s)=F_{x}(s)
$$

Setting $s=j \omega$, then

$$
X(j \omega)=\frac{F_{X}(j \omega)}{\left(k-m \omega^{2}\right)+j \omega c}
$$

That is the frequency-response equation of second order mechanical system.

Comparing (16) with (19), closed loop stiffness of the system is signed as $k_{x x}$ corresponding to $k$, and closed loop damping is signed as $c_{x x}$ corresponding to $c$.

$$
\begin{aligned}
& k_{x x}=k_{x x 0}+k_{x i 0} R_{e}[G(j \omega)] \\
& c_{x x}=k_{x i 0} I_{m}[G(j \omega)] / \omega
\end{aligned}
$$

So equation (6) is turned into

$$
\Delta F_{x}=k_{x x} x+c_{x x} \dot{x}
$$

\section{PID CONTROLLER AND ITS PARAMETER SET- TINGS}

Stiffness and damping of the system are closely related to parameters of controller. As mentioned, transfer function of maglev control system is composed of $G_{s}(s), G_{c}(s)$ and $G_{p}(s)$.

The transfer function of sensor $G_{s}=A_{s} /\left(1+T_{s} s\right)$, where $A_{s}$ is the gain, bandwidth is $5 \mathrm{kHz}, T_{s}=3.18 \times 10^{-5}$ is the decay time constant.

The transfer function of controller is composed of several parts: addition, second order filter, proportion, integral and differential.

$G_{c}=G_{p 1} G_{p 2} G_{p 3} G_{i} G_{d}$

Transfer function of addition circuit is shown as following

$G_{P 1}=\frac{A_{p 1}}{1+T_{p 1} s}$

Where, $A_{p 1}$ is the gain, $T_{p 1}$ is decay time constant.

Transfer function of second order filter is shown as following

$G_{p 2}=\frac{1}{1+T_{p 2} s+T_{p 22} s^{2}}$ 

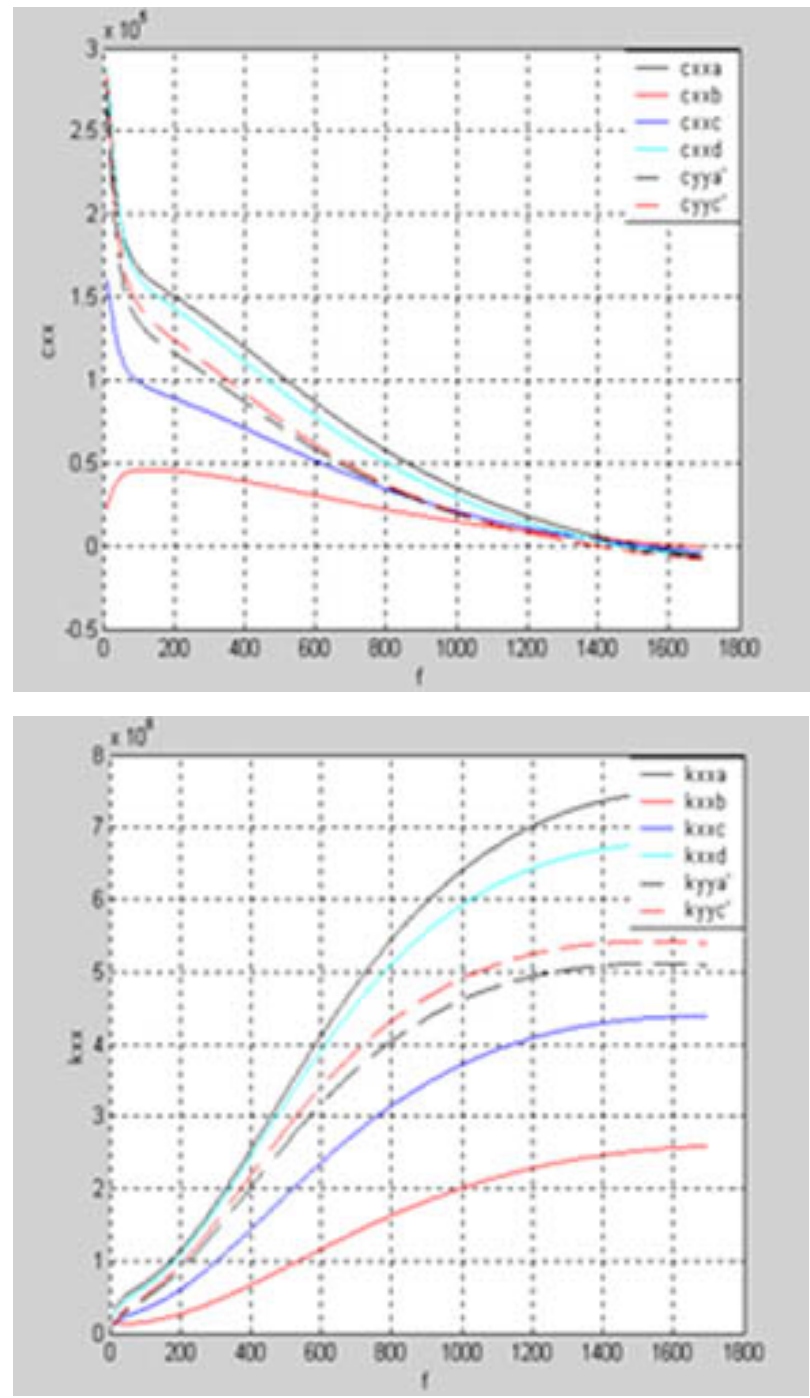

Fig. (5). Stiffness and damping curves corresponding to the first group of parameters.

Where, $T_{p 2}$ and $T_{p 22}$ are decay time constants.

Transfer function of proportion circuit is shown as following

$G_{p 3}=A_{p 3}$ ing

Transfer function of integral circuit is shown as follow-

$G_{i}=A_{i} \frac{1+T_{i 2} s}{1+T_{i 1} s}$

Where, $A_{i}$ is the gain, $T_{i 1}, T_{i 2}$ are decay time constant.

Transfer function of differential circuit is shown as following

$G_{d}=\frac{1+\left(R_{120} C_{106}+T_{d}\right) s}{1+\left(R_{120} C_{106}+\varepsilon T_{d}\right) s}$

Where, $T_{d}$ is decay time constant, $\varepsilon=R_{119} /\left(R_{118}+R_{119}\right), R$ and $C$ are resistors and capacitors in specific circuit.
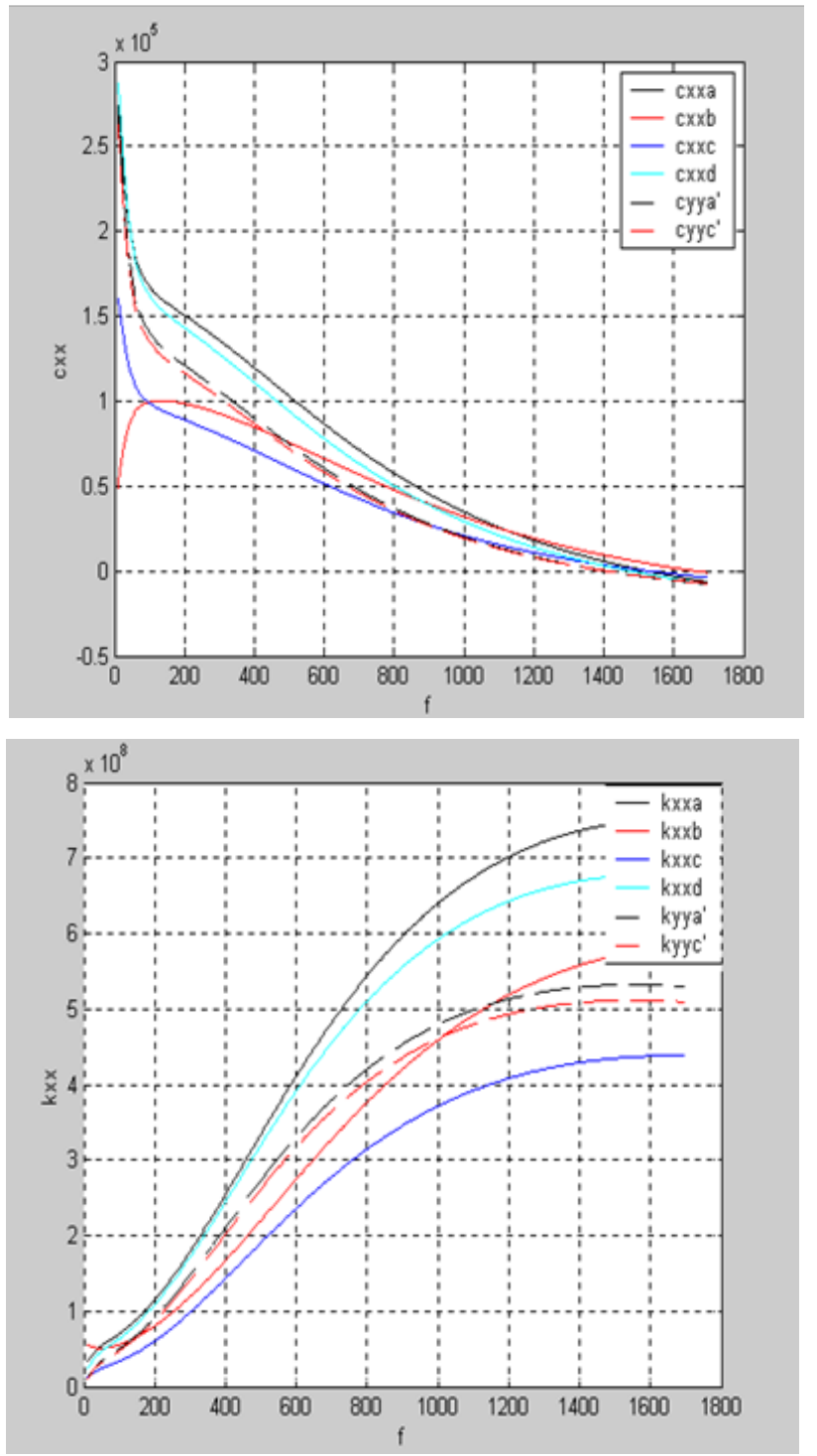

Fig. (6). Stiffness and damping curves corresponding to the second group of parameters.

Transfer function of power amplifier is shown as following

$G_{p}=A_{m} \frac{\left(1+T_{m 1} s\right)\left(1+T_{m 2} s\right)}{\left(1+T_{m 1} s\right)\left(1+T_{m 2} s\right)\left(1+T_{m 3} s\right)+A_{m} R_{L 21} T_{m 4} s}$

Where, $A_{m}$ is the gain, $T_{m 1}, T_{m 2}, T_{m 3}, T_{m 4}$ are decay time constants.

Transfer function of total controller can be obtained by putting the transfer function of each part and related parameter into $G(s)$.

\section{EXPERIMENT RESULTS}

Based on system model built, for maglev linear feed motion guide, its mechanical properties are calculated by solving the proper equations using MATLAB. As computing eigenvalues, a $N$ degree algebraic equation requires to be solved, which require heavy computational work when $N$ is 
Table 1. The first group of control parameters.

\begin{tabular}{|c|c|c|c|c|}
\hline Channel & $\boldsymbol{W} / \boldsymbol{k} \boldsymbol{\Omega}$ & $\boldsymbol{W p} / \boldsymbol{k} \boldsymbol{\Omega}$ & $\boldsymbol{W i} / \boldsymbol{k}$ & 4.72 \\
\hline \hline 1 & 6.79 & 0.750 & 5.18 & 3.94 \\
\hline 2 & 9.44 & 0.690 & 4.37 & 3.73 \\
\hline 3 & 3.80 & 0.690 & 3.13 & 4.79 \\
\hline 4 & 6.56 & 0.950 & 6.58 & 6.12 \\
\hline 5 & 3.64 & 0.756 & 6.18 & 6.12 \\
\hline
\end{tabular}

Table 2. The second group of control parameters.

\begin{tabular}{|c|c|c|c|c|}
\hline 1 & 6.79 & 0.750 & 3.94 & 4.72 \\
\hline 3 & 3.80 & 0.690 & 3.87 & 4.37 \\
\hline 5 & 3.60 & 0.780 & 6.12 & 6.18 \\
\hline 6 & 3.65 & 0.760 & 6.32 & 6.18 \\
\hline
\end{tabular}

very big. Given two groups of different parameters, dynamic characteristic is calculated as maglev feed motion guide is in ideal operating condition. Comparing dynamic characteristics in different parameters, theoretical researches and simulations are done for structure and controller design of maglev linear feed motion guide. Stiffness and damping curves are respectively shown as in Fig. (5) and Fig. (6) based on two groups of control parameters as shown Table (1 and 2 ) using MATLAB [8-10].

As seen from Fig. (6), the system only has first order critical frequency, and resonant unstable frequency is $1406 \mathrm{~Hz}$ by using the first group of parameters. In this case, all real parts of the eigenvalues are negative, so the system is stable. As seen from Fig. (7), the system has no critical vibration, and resonant unstable frequency is $1400 \mathrm{~Hz}$ by using the second group of parameters. In this case, all real parts of the eigenvalues are negative, so the system is stable, and stiffness and damping of the system is much larger than in the first case.

\section{CONCLUSION}

The stiffness and damping of maglev supporting system depends on not only its structure parameters but also the controller parameters. Channel control of system determines that it has multiple different characteristic curves of stiffness and damping, and stability of system requires that each channel parameter of control system is generally different and the eigenvalues must be negative. Stiffness and damping of system has a big influence, it does not only change the values but also the order of critical frequency. Part of maximum vibration amplitude in the system is the position where electromagnet is installed, and the region of smaller vibration is the center of the guide-way, which is very important for feed motion guide because various components, such as fixture, spindle, etc, are installed there because small vibration can improve machining precision. By appropriately adjusting parameters maglev feed motion guide system can reach an optimal dynamic performance.

\section{CONFLICT OF INTEREST}

The author confirm that this article content has no conflict of interest.

\section{ACKNOWLEDGEMENTS}

We acknowledge the help of Bing Zhang and Wensheng Huang in research. We also thank the anonymous reviewers for their numerous helpful suggestions. The work was supported by the National Nature Science Foundation of China (No. 51175052) and Applied Basic Research Programs of Changzhou City (No. CJ20130014), also supported by Scientific Research Fund of Changzhou Institute of Technology (No.YN1216), respectively. 


\section{REFERENCES}

[1] Z. Jiansheng, Z. Gang, W. Guoqing, W. Xiping and W. Minggui, "The Research of Guide Supported with AMB", Machine Building \& Automation, vol. 33(6), pp.127-130, Nov. 2004.

[2] H. Dezhong, "The desigen of magnetic suspension slideway", Modular Machine Tool \& Automatic Manufacturing Technique, no. 12, pp. 77-80, Jan. 2004.

[3] C. Kim, K. Kim,, J. Yu, C. Han-Wook, "Dynamic performance evaluation of 5-dof magnetic levitation and guidance device by using equivalent magnetic circuit model", IEEE Transactions on Magnetics, vol. 49(7), pp. 4156-4159, 2013.

[4] Q. Bin, D. Liming, Z. Haibo, H. Yi and D. Ji'an, "nonlinear modeling and analysis of magnetic force in precision magnetic levitation system", Journal of Huaqiao University (Natural Science), vol. 30, no. 1, pp. 22-26, Jan. 2009.

[5] W. Qiang, Q. Yongming, M. Suyang, Y. Ji and L. Ping, "The stiffness optimization analysis of magnetically suspended table", Manufacturing Automation, vol. 35, no. 7, pp. 52-55, July 2013.
[6] W. K. S. Khoo, K. Kalita, S. D. Garvey, R. J. Hill-Cottingham, D. Rodger, and J. F. Eastham, "Active axial-magnetomotive force parallel-airgap serial flux magnetic bearings," IEEE Transactions On Magnetics, vol. 46, no. 7, pp. 2596-2602, July 2010.

[7] Z. Jing and X. Lin, "Fuzzy adaptive PID control in magnetic levitation system," Journal of Computer Applications, vol. 29, no. 6, pp. 329-334, June 2009.

[8] L. Wenguang, H. Junfeng, and L. Hongli, "Efficient Simulation of Fuzzy Control System Based on MATLAB (SIMULINK)," Computer Simulation, vol. 18, no. 3, pp. 15-16, June 2001.

[9] W. Xiaoling and Z. Guangming, "Magnetic Levitating Bearing Control System Simulation Based on Variable Universe Fuzzy PID Algorithm," Instrument Technique and Sensor, no. 12, pp. 144-147, December 2012.

[10] L. Jinkun, Editor, Advanced PID Control and MATLAB Simulation (1st ed.), Publishing House of Electronics Industry, Beijing, 2003.

(C) Zheng et al.; Licensee Bentham Open.

This is an open access article licensed under the terms of the Creative Commons Attribution Non-Commercial License (http://creativecommons.org/licenses/by-nc/3.0/) which permits unrestricted, non-commercial use, distribution and reproduction in any medium, provided the work is properly cited. 